Apidologie, 1980, 11 (4), 385-400.

\title{
APIS MELLIFERA ADAMI (n. ssp.), DIE KRETISCHE BIENE
}

\author{
Apis mellifera adami \\ (n. ssp.), abeille crétoise
}

\author{
Friedrich RUTTNER \\ Institut für Bienenkunde \\ D - 6370 Oberursel \\ R.F.A.
}

SUMMARY

APIS MELLIFERA ADAMI (n. ssp.). THE CREATEAN BEE

Elaborating on an earlier preliminary communication (1975), the bee of the island of Crete is described as a separate geographic race based on an examination of representative material. This race is named after Brother Adam who collected the first samples. The race is clearly differentiated from the other known races of bees by a large number of morphological characters and biological traits. The Cretean bee is very similar to bees of Western Anatolia and the East Egean Islands (Dodekanes).

Multivariate analysis shows the similarities of $A . m$. adami to $A . m$. syriaca ButTEL-REEPEN and to A. m. intermissa ButTel-ReEpen but not to European races as $A$. m. carnica PollmanN.

\section{ZUSAMMENFASSUNG}

In Erweiterung einer früheren vorläufigen Mitteilung (1975) wird an Hand eines repräsentativen Materials die Biene von Kreta als eigene geographische Rasse beschrieben, die sich durch eine grosse Anzahl von morphologischen Merkmalen und von biologischen Eigenschaften von allen anderen bekannten Rassen klar abgrenzen lässt. Die Kretische Biene besitzt grosse Ähnlichkeit mit der Biene von Westanatolien und von den ostägäischen Inseln (Dodekanes).

Nach den Ergebnissen von Multivariatanalysen weist diese ganze Gruppe Beziehungen zu A. m. syriaca ButTEL-REEPEN und $A$. $m$. intermissa BUTTEL-REEPEN auch, jedoch kaum zu europäischen Rassen wie A. m. carnica Pollmann. 


\section{EINLEITUNG}

Vor einigen Jahren wurde in Form einer vorläufigen Mitteilung über eine sehr charakteristische Bienenrasse von der Insel Kreta berichtet, für die die Bezeichnung " adami " vorgeschlagen wurde, zu Ehren des bekannten Züchters und Erforschers der Bienenrassen, BRUDER ADAM, Buckfast (RUTTNER, 1975). Inzwischen konnten weitere Bienenproben von Kreta und von anderen griechischen Inseln untersucht und das ganze Material einer eingehenden statistischen Analyse unterzogen werden, so dass es heute möglich ist, eine quantitative Beschreibung der Merkmale dieser Rasse vorzulegen. Ausserdem wurde das Verhalten dieser Biene in ihrem natürlichen Habitat sowie an Hand von vier Völkern im Taunusgebiet beobachtet, so dass auch einige Angaben zur Biologie dieser Biene möglich sind.

\section{MATERIAL UND METHODEN}

Folgende Bienenproben standen für die Untersuchung zur Verfügung :

4 Proben gesammelt 1957 von Bruder ADAM in Chania und Heraklion (Proben Nr. 146, 147, 259, 260).

12 Proben gesammelt 1974 von Herrn TsiKalas, Sisses in Ost-, Mittel- und Westkreta (Proben Nr. 594-605).

8 Proben, 1976 vom Autor an verschiedenen Stellen der Insel gesammelt (Proben Nr. 687-689, 691695).

Das sind 24 Proben Arbeitsbienen insgesamt.

Ausserdem standen 8 Drohnenproben aus verschiedenen Teilen der Insel zur Verfügung (Proben-Nr. $622-624,687,688,691,693,695)$.

Dr. MaLitzKy (Lunz am See) brachte von seinen entomologischen Sammelreisen Bienenproben von vier Jonischen Inseln (Zakinthos, Kefalonia, Lefkas und Kerkira) und von Kithira, sowie von Karpathos (gesammelt von Dr. PIEPER, Kiel), von Rhodos (Dodekanes), und von Chios und Lesbos mit (die vier letztgenannten Inseln werden hier als « Ostägäische Inseln » bezeichnet).

Im Gegensatz zu den Bienenproben aus Kreta, die in der üblichen Weise aus Völkern entnommen worden waren, wurden diese Bienen einzeln von Blüten abgefangen. Die Bienen einer Probe stammten also zwar aus derselben lokalen Population, aber nicht aus demselben Volk. Ausserdem muss bei der Beurteilung des Haarkleides berücksichtigt werden, dass es sich ausschliesslich um Flugbienen handelt.

Die Konservierung und Präparierung der Bienen, und die Messung und statistische Bearbeitung der Merkmale erfolgt nach einer Standardmethode (RuTTNER et al. 1978).

Eine Bienenprobe umfasst im allgemeinen 20 Bienen. Für die 24 Proben aus Kreta wurden also 480 Bienen biometrisch bearbeitet, mit 38 Einzelmessungen pro Biene. Aus den 20 Werten einer Probe wurde für jedes Merkmal der Mittelwert berechnet. Die Mittelwerte der 24 Proben aus Kreta bildeten die Grundlage für die Berechnung des Mittelwertes $(\bar{x})$ und der Standardabweichung $(s)$ der Bienenpopulation von Kreta. Jeder dieser Werte beruht also auf 480 Einzelmessungen.

In gleicher Weise wurden die Mittelwerte für die Gruppe « Jonische Inseln " (6 Proben mit 96 Bienen) und die " Ostägäische Inseln » (4 Proben mit 51 Bienen) gebildet. 


\section{Morphometrische Abgrenzung}

Um einen Überblick über die biometrische Gesamtstruktur der Spezies Apis mellifera L. zu gewinnen, wurden die Daten von 33 Merkmalen bei 404 Proben nach der Methode der "principal components" einer globalen Analyse unterzogen*. Auf einer graphischen Darstellung der in dieser Analyse gewonnenen Faktoren 1 und 2 (welche den Hauptanteil der Gesamtvariabilität umfassen) in einem Koordinatensystem bilden die Punkte, welche die Proben aus Kreta repräsentieren, ein relativ enges Feld (cluster) an der Basis des " $\mathbf{M}$ 》-Astes der Y-förmigen Struktur der Species (RUTTNER et al. 1978, RUTTNER 1977). Der M-Ast wird vor allem gebildet durch die Rassenkette intermissa-iberica-mellifica. Das Feld der nordafrikanischen Intermissa überdeckt auf dieser Darstellung teilweise das Feld der adami. Wie ein Vergleich der Werte von adami (Tab. 1 dieser Arbeit) mit einigen Daten von intermissa (Tab. 1 in RuTTNER 1975) zeigt, kommt dies daher, dass beide Rassen ein etwa gleich grosses (Tergit $3+4$ ) und gleich geformtes ("Schlankheitsindex" von Sternit 6) Abdomen besitzen und in verschiedenen anderen Merkmalen (Rüssel- und Haarlänge, Cubitalindex, verschiedene Aderwinkel) sehr ähnlich sind. In anderen Merkmalen hingegen, wie Pigmentierung und Filzbindenbehaarung, bestehen erhebliche Unterschiede, auch ist die adami langbeiniger und kurzflügliger wie die intermissa. In einer Spezialanalyse ist es nicht schwierig, diese beiden Rassen biometrisch voneinander zu trennen.

Das adami-Feld auf dieser Darstellung überdeckt ferner in einem kleinen Abschnitt das lang gezogene, gewinkelte Feld, das von den Bienenproben aus Anatolien eingenommen wird. In einer geographischen Spezialdarstellung von Proben aus dem östlichen Mittelmeergebiet (ohne Ägypten) wird sehr deutlich, dass die Bienenproben aus Westanatolien (Izmir) z. T. ganz nahe am adami-Feld liegen (Abb. 1). Dasselbe gilt für die Proben von den Anatolien westlich vorgelagerten ägäischen Inseln und des Dodekanes (s. auch Tab. 1). In der Tat geht aus dieser Tabelle sehr deutlich hervor, dass fast alle Merkmale der ostägäischen Biene gleich oder sehr ähnlich sind wie die der Biene aus Kreta. Auf der anderen Seite ist gegenüber den " ionischen " Bienen - die gleichgesetzt werden können mit den Bienen des griechischen Festlandes, die zum Carnica-Komplex gehören - in allen 22 angeführten Merkmalen ein sehr deutlicher Unterschied festzustellen. Dementsprechend ergibt sich auf Abb. $1 b$ eine sehr deutliche Abgrenzung des adami-Feldes von dem Areal, das von der griechischen (und auch der italienischen) Biene eingenommen wird. Auch gegenüber den beiden anderen

- Die Hauptkomponenten-Analyse ermöglicht es, sogenannte synthetische Variablen (" principal components ") zu konstruieren, die mit allen gemessenen Variablen möglichst hoch korrelieren und damit die in den Variablenwerten enthaltene Information möglichst gut wiedergeben. Dadurch wird die Zahl der Daten so reduziert, dass die Information, die uns eine grosse Anzahl von gemessenen Variablen bietet, in zwei- oder dreidimensionaler Darstellung wiederzugeben ist. 


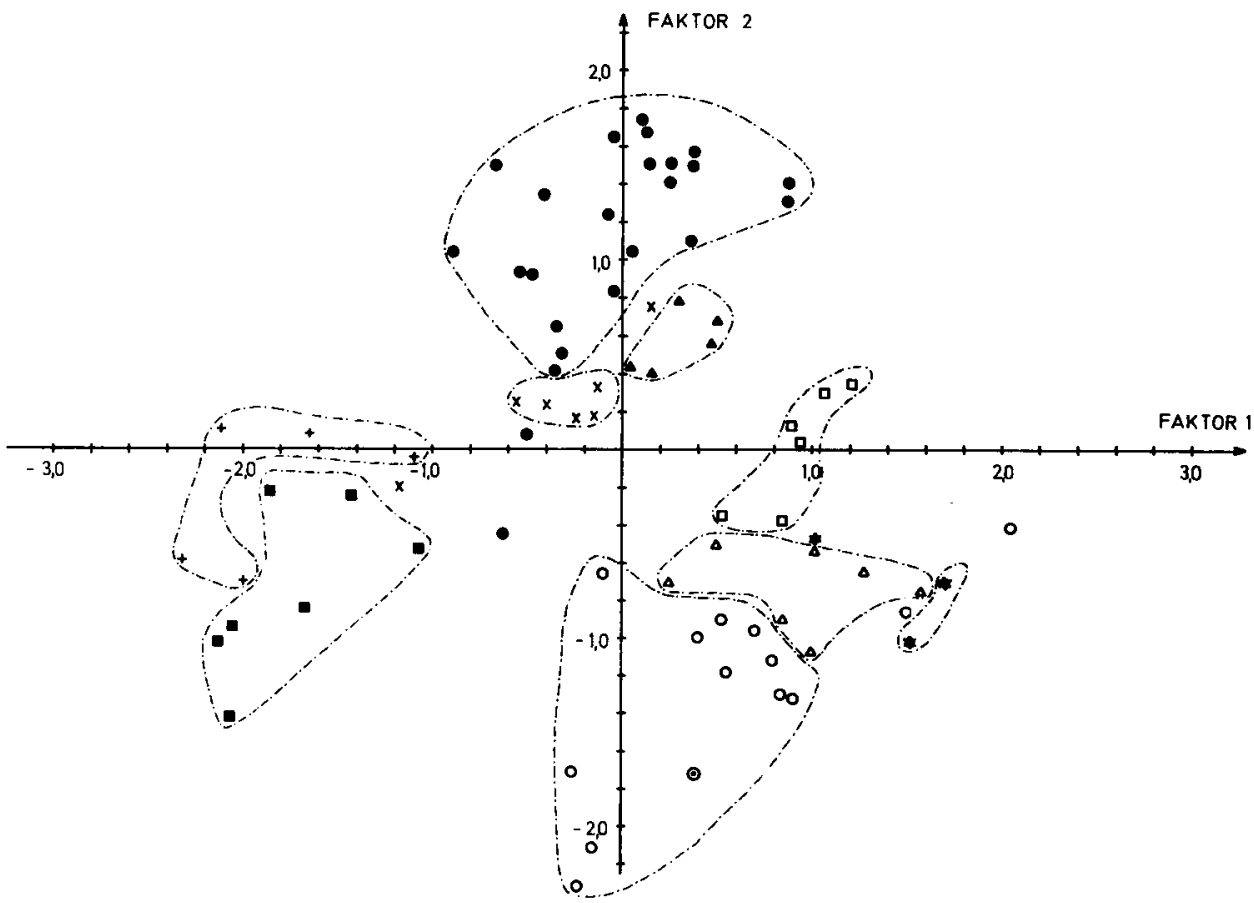

ABB. 1. - Graphische Darstellung des Ergebnisses einer Faktorenanalyse an 77 Bienenproben und 34 Merkmalen aus dem ostmediterranen Raum. Faktor 1 (Abszisse : 25,6\% der Gesamtvariabilität) gegen Faktor 2 (18,7\% der Gesamtvariabilität). Deutliche Trennung in drei Komplexe : Kreta mit Westanatolien und den griechischen Inseln, syriaca u. cypria und intermissa.
o Algerien
Algérie
D Ionische Inseln Iles ioniques
- Kreta
Crète
- Malta
Malte
- Ost-Agäis
Mer Égée orientale
$\triangle$ Sizilien
Sicile
+ Syrien
Syrie
- Tunesien
Tunisie
$x$ West-Anatolien
Anatolie occidentale
- Zypern
Chypre

Fig. 1. - Représentation graphique de l'analyse factorielle portant sur 77 échantillons d'abeilles provenant de la Méditerranée orientale et sur 34 caractères. Facteur 1 (abscisse : $25,6 \%$ de la variabilité totale) contre facteur $2(18,7 \%$ de la variabilité totale). Séparation nette en 3 complexes : la Crète avec l'Anatolie occidentale et les îles grecques, syriaca et cypria et intermissa.

mediterranen Inselrassen, der zyprischen und der sizilianischen Biene, ist die KretaBiene einwandfrei getrennt, ebenso wie von der syrischen Biene in Israel, Jordanien und Libanon.

Die Gegenüberstellung auf Tab. 1 ergibt die morphologische Charakterisierung von A. m. adami. 
2. Beschreibung der Unterart Apis mellifera adami n. ssp.

Holotypus : Probe mit 20 Arbeiterinnen und 20 Drohnen, Forschungsinstitut Senckenberg Frankfurt/Main, Marathos b. Heraklion, Kreta, 18-4-1976 (Pr. Nr. 687), leg. F. RUTTNER.

Paratypen : 23 Proben aus Kreta im Institut f. Bienenkunde, Oberursel.

Ableitung des Namens : Bruder Adam KeHrLe, Benediktinerkloster, St. Mary's Abbey, Buckfast, Devon, England, der sich durch eine umfangreiche Sammlung von autochthonen Bienenproben aus dem mediterranen Gebiet und die Untersuchung der biologischen Eigenschaften dieser Formen sowie ihrer ökonomischen Bedeutung verdient gemacht hat.

Beschreibung (Tab. 1; die Charakterisierung ergibt sich aus dem Vergleich von Spalte 3 mit Spalte 7).

\section{1. Arbeiterinnen}

Bienen von mittlerer Körpergrösse (Merkmal 44), relativ langen Beinen (Merkmal 43), aber kurzen und auch schmalen Flügeln (Merkmal 21, 22). Besonders charakteristisch sind einige andere Körperproportionen: Während Femur und besonders Tibia wesentlich länger sind als bei der griechischen Biene, ist der Metatarsus kürzer (Merkmal 8); die Typisierung als « langbeinig " wird dadurch zwar nicht geändert, aber zusammen mit einem erhöhten Querdurchmesser der Metatarsen (Merkmal 9) ergeben sich ausgesprochen breite Fersen (Metarsalindex 56,38 $=$ Merkmal 45). Dasselbe Merkmal wurde schon von GersTÄCKer (1862) zur Charakterisierung der kaukasischen $A$. $m$. remipes (GERSTÄCKER) (= ruderfüssig) verwendet. Im selben Sinne sind auch die Proportionen des Abdomens einzuordnen. In der Spezies A. mellifera L. gibt es "schlanke" und "breite" Formen, sehr deutlich schon am Gesamthabitus erkennbar und quantitativ durch den "Schlankheitsindex " (Merkmal 47) dem Verhältnis Länge : Breite des herzförmigen letzten (6.) Sternits erfassbar. Ein weiteres Charakteristikum ist der besonders grosse Abstand zwischen beiden Wachsspiegeln auf Sternit 3 (Merkmal 18). Gemeinsam mit den Rassen intermissa, iberica und mellifera gehört $A$. m. adami zu den "breiten" Formen. Die Rüssellänge liegt mit $6,460 \mathrm{~mm}$ etwa in mittleren Bereich - aber deutlich tiefer als bei den griechischen Bienen. Das Überhaar des Abdomens ist kurz $(0,30 \mathrm{~mm})$, so wie bei allen übrigen Rassen der Region. Die Filzbinden sind breit und dicht, sie tragen sehr wesentlich zum Habitus der Biene bei (Merkmale 1 u. 49).

Nach der Pigmentierung des Abdomens ist die Kretische Biene eine ausgesprochen gelbe Biene. Der mittlere Skalenwert von 5.66 auf dem 3. Tergit der 25 Proben bedeutet einen durchgehenden, deutlichen gelben Streifen auf diesem Tergit. Der mittlere Farbwert des 2 . Tergits ist gleichgross $(5,66)$, während das 4 . Tergit relativ 


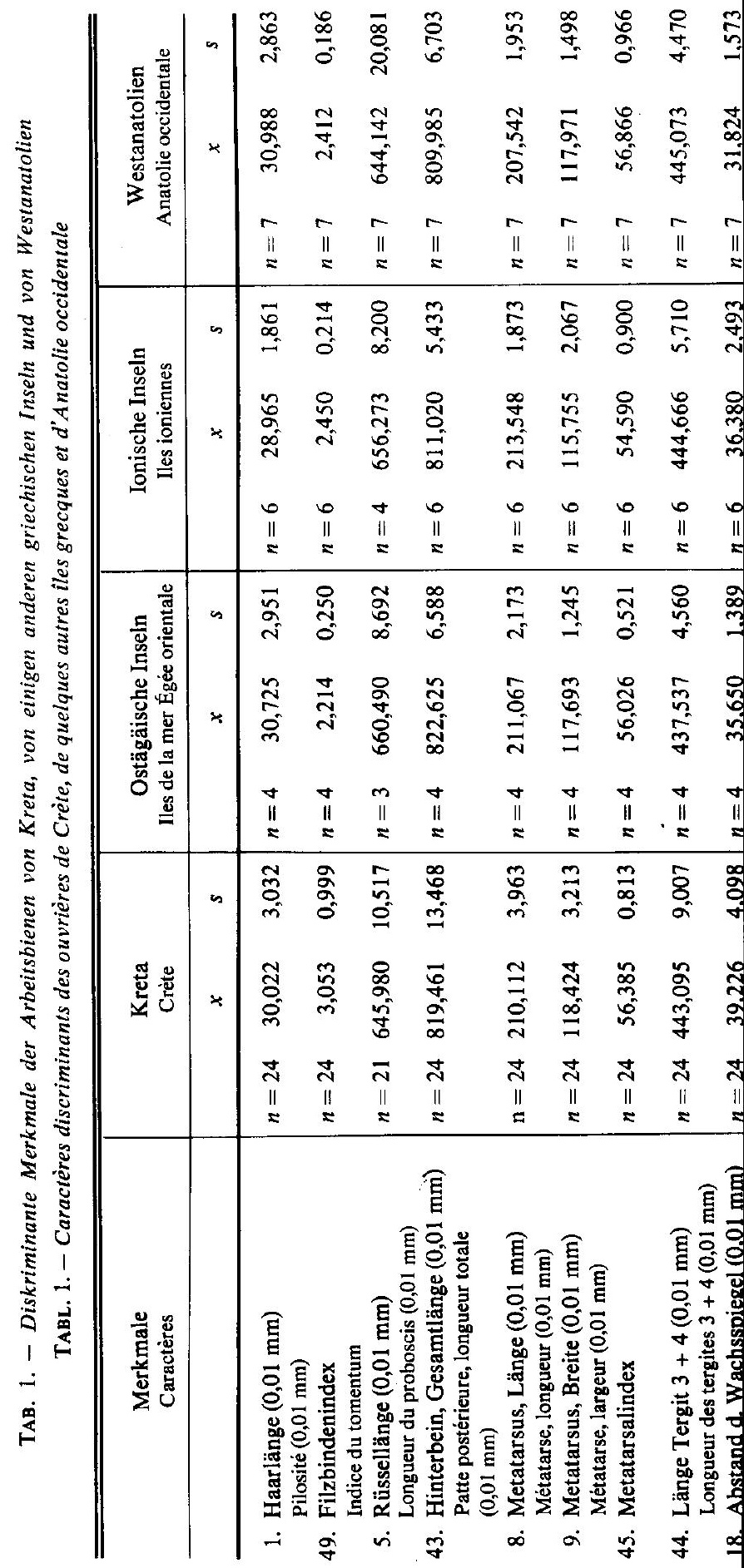


kleine gelbe Ecken aufweist (Farbwert 2,50). Die Biene hat also im Mittel auf den beiden vordersten der zur gänze sichtbaren Tergite gelbe Ringe. Bemerkenswert ist die grosse Variabilität der Pigmentierung. Nach dem allgemeinen optischen Eindruck wirkt eine Biene " dunkel ", wenn ihr Farbwert auf Tergit 3 unter 5,0 liegt, " gelb " bei Werten über 5,0. Es zeigt sich, dass nur ein kleiner Teil der Völker einigermassen einheitlich "gelb" ist, die Mittelwerte der Farbskala liegen dann zwischen 6,0 und 8,0. Etwa die Hälfte der Völker enthalten "gelbe " und "dunkle " Bienen zu etwa gleichen Teilen.

Meist liegt das Mittel des Farbwertes zwischen 4,5 und 5,5. Da die gelbe Pigmentierung stärker auffällt, wirken auch diese Völker im Gesamteindruck " gelb ". Es gibt aber auch Völker, die "dunkel " aussehen, mit einem mittleren Farbwert von weniger als 4,50 auf Tergit 3 (im vorliegenden Material 3 Völker von 24).

Im Gegensatz zu der überwiegend gelben Pigmentierung der vorderen Abdominaltergite ist das Scutellum überwiegend dunkel (Farbwert 2,34, Nr. 23), d.h. es hat schwache diffuse Aufhellungen oder - häufiger - zarte gelbe Streifen an den Rändern. Aber auch hier ist die Variabilität beträchtlich; zwei Völker sind im Scutellum " gelb " (mittlerer Farbwert über 5,0), 13 Völker " dunkel " (Farbwert unter 2,0). Noch geringer sind die Aufhellungen bei den kleinen Skleriten seitlich und hinter dem Scutellum (Meta- und Mesotergum).

Besonders charakteristisch für die Kretische Biene ist der Cubitalindex, der mit einem Mittelwert von 1,891 nahe dem unteren Ende der Variationsbreite der bekannten Bienenrassen liegt. Die Variation zwischen den Völkern ist gering, die Mittelwerte der Völker liegen zwischen 1.536 und 2.149. Von den in der Standardbiometrie gemessenen 11 Winkeln im Geäder des Vorderflügels wurde in Tab. 1 die sechs aufgenommen $(\mathrm{Nr}$. 31-35-40), welche gegenüber den benachbarten Rassen die deutlichsten Unterschiede zeigen. Sie tragen in beträchtlichem Masse zur Diskriminanz bei.

\subsection{Drohnen}

Zur Charakterisierung der Drohnen von $A$. m. adami wurden 15 Merkmale herangezogen (Tab. 2). Hinsichtlich der Grösse liegen auch die Drohnen im mittleren Bereich der Gesamtvariabilität der Art. Gegenüber der grossen Subspecies Carnica (aus Jugoslawien) sind alle Grössenmasse deutlich geringer (Tab. 2, Nr. 1-3, 5, 6). Der Metatarsus ist auch bei den Drohnen relativ breit.

Eigenartigerweise ist die Pigmentierung der Drohnen im Gegensatz zu den Arbeiterinnen einheitlich dunkel. Der mittlere Skalenwert von 2,354 entspricht kleinen gelben "Inseln " in der Nähe der Stigmen des 3. Tergits, bei sonst ganz dunkler Pigmentierung. Auch das Scutellum ist einheitlich dunkel. Damit unterscheidet sich diese Rasse sehr deutlich z.B. von der italienischen ligustica, bei der Arbeiterinnen und Drohnen " gelb * sind.

Auch die Königinnen sind immer am ganzen Körper dunkel pigmentiert. 
TAB. 2. - Merkmale von Drohnen von Kreta (Mittelwerte aus je 8 Proben zu je 20 Tieren)

TABL. 2. - Caractères des mâles de Crète (moyenne de 8 échantillons de 20 insectes chacun).

\begin{tabular}{|c|c|c|c|c|}
\hline $\begin{array}{l}\text { Merkmal } \\
\text { Caractère }\end{array}$ & \multicolumn{2}{|c|}{$\begin{array}{l}\text { Kreta } \\
\text { Crète }\end{array}$} & \multicolumn{2}{|c|}{ Jugoslawien } \\
\hline $\begin{array}{l}\text { 43. Hinterbein, Gesamtlänge }(0,01 \mathrm{~mm}) \\
\text { Patte postérieure, longueur totale }(0,01 \mathrm{~mm})\end{array}$ & 960,491 & 17,263 & 985,189 & 27,275 \\
\hline $\begin{array}{l}\text { 8. Metatarsus Länge }(0,01 \mathrm{~mm}) \\
\text { Métatarse, longueur }(0,01 \mathrm{~mm})\end{array}$ & 243,306 & 5,275 & 258,702 & 8,468 \\
\hline $\begin{array}{l}\text { 9. Metatarsus, Breite }(0,01 \mathrm{~mm}) \\
\text { Métatarse, largeur }(0,01 \mathrm{~mm})\end{array}$ & 121,046 & 2,711 & 123,326 & 6,123 \\
\hline $\begin{array}{l}\text { 45. Metatarsal-Index } \\
\text { Indice métatarsal }\end{array}$ & 49,788 & 1,282 & 47,695 & 2,253 \\
\hline $\begin{array}{l}\text { 21. Flügellänge }(0,01 \mathrm{~mm}) \\
\text { Longueur de l'aile }(0,01 \mathrm{~mm})\end{array}$ & 1169,902 & 16,536 & 1211,556 & 27,859 \\
\hline $\begin{array}{l}\text { 22. Flügelbreite }(0,01 \mathrm{~mm}) \\
\text { Largeur de l'aile }(0,01 \mathrm{~mm})\end{array}$ & 371,133 & 7,251 & 396,764 & 10,466 \\
\hline $\begin{array}{l}\text { 11. Farbwert auf T } 3 \text { (Skalenwerte) } \\
\text { Coloration sur le T } 3 \text { (valeurs dans l'échelle) }\end{array}$ & 2,354 & 0,620 & $1,380^{*}$ & 0,970 \\
\hline $\begin{array}{l}\text { 23. Farbwert Scutellum } \\
\text { Coloration du scutellum }\end{array}$ & 0,878 & 0,696 & 1,292 & 0,693 \\
\hline $\begin{array}{l}\text { 48. Cubitalindex } \\
\text { Indice cubital }\end{array}$ & 1,308 & 0,180 & 1,976 & 0,288 \\
\hline $\begin{array}{l}\text { 31. Aderwinkel A } 4 \\
\text { Angle des nervures A } 4\end{array}$ & 28,546 & 1,448 & 26,038 & 2,209 \\
\hline $\begin{array}{l}\text { 32. Aderwinkel B } 4 \\
\text { Angle des nervures B } 4\end{array}$ & 107,813 & 4,374 & 114,846 & 5,886 \\
\hline $\begin{array}{l}\text { 33. Aderwinkel D } 7 \\
\text { Angle des nervures D } 7\end{array}$ & 110,296 & 2,451 & 106,239 & 2,695 \\
\hline $\begin{array}{l}\text { 34. Aderwinkel E } 9 \\
\text { Angle des nervures E } 9\end{array}$ & 18,946 & 0,794 & 22,220 & 1,764 \\
\hline $\begin{array}{l}\text { 35. Aderwinkel G } 18 \\
\text { Angle des nervures G } 18\end{array}$ & 93,100 & 1,542 & 89,327 & 2,746 \\
\hline $\begin{array}{l}\text { 40. Aderwinkel N } 23 \\
\text { Angle des nervures N } 23\end{array}$ & 85,569 & 2,549 & 86,545 & 3,244 \\
\hline
\end{tabular}

* Farbwert auf T 2.

Coloration sur le T 2.

Die Farbe der Behaarung am Thorax der Drohnen, bestimmt nach der Skala von Goetze (RuTTNER, 1979) ist " lehmgrau bzw. etwas dunkler ".

Der Cubitalindex ist sehr niedrig, vergleichbar mit dem von der Rasse mellifera. Bei einer Probe (Nr. 691) liegt der Mittelwert sogar unter 1,0 (0,965), d.h. die Strecke $b$ ist länger als die Strecke $a$.

Die Verschiebung der sechs in der Tabelle angeführten Aderwinkel gegenüber der Rasse carnica ist deutlich; sie erfolgt im selben Sinne wie bei den Arbeiterinnen.

Der Vergleich der beiden Probengruppen ergibt eine deutlich niedrigere Standardabweichung für die Proben als Kreta. Das spricht für eine hohe Einheitlichkeit der Inselpopulation. 


\section{Biologie und Eigenschaften von wirtschaftlicher Bedeutung}

Kreta ist ein Bienenland von der prähistorischen Zeit bis zum heutigen Tag (RUTTNER, 1977). Mit 10 Bienenvölkern pro Quadratkilometer gehört sie zu den am dichtesten mit Bienen besetzten Gebieten der Erde (RUTTNER, 1976).

Die Insel Kreta liegt im mediterranen Klima- und Florenbereich. Der allgemeinen Tendenz entsprechend ist der Osten der Insel deutlich trockener als der Westen.

An den Küsten gibt es einen Macchiengürtel, dessen Florenelemente (Erica arborea, Cistus, Salvia, Lavandula u.a.) ihre Blütezeit während der Winter- und Frühjahrsmonate haben. In den Küstenebenen nimmt die Kultur von Citrusfrüchten von Jahr zu Jahr zu. Die wichtigste Trachtquelle für die Bienen aber sind die ausgedehnten Thymusbestände auf den steinigen Hängen, deren Blütezeit (je nach Meereshöhe) in den Monaten Juli und August liegt.

Dem Trachtangebot entsprechend zeigt der Brutrhythmus der Kretischen Biene einen ausgesprochen mediterranen Typus. Bruttätigkeit wird den ganzen Winter über aufrecht erhalten, mit 2-3 Brutkreisen von etwa $15 \mathrm{~cm}$ Durchmesser. Ab Februar erfolgt ein steiler Anstieg der Bruttätigkeit, die im April/Mai mit 14-18 Langstrothwaben Brut ihren Höhepunkt erreicht, dadurch kommen die Völker rasch zu einer erheblichen Volksstärke. Ab Mitte Mai gibt es eine Trachtpause von etwa 6 Wochen Dauer. In dieser Zeit wird der Brutumfang verringert, es besteht ein starker Bienenüberschuss. Ein zweiter, aber nicht mehr so starker Brutanstieg im Juli führt zu der charakteristischen zweigipfligen Brutkurve der Mittelmeerländer.

Die Kretische Biene ist eine schwarmfreudige Rasse. Ist einmal eine bestimmte Volksstärke erreicht, dann bereitet sich fast jedes Volk zum Schwärmen vor. Die Zahl und Anordnung der Weiselzellen ist ähnlich wie bei orientalisch-nordafrikanischen Rassen - in einer engen Reihe am Wabenrand, 60-200 Stück.

Eigenartig ist das Temperament der Biene. Auf der Wabe ist das Verhalten ausgesprochen ruhig. Bei Entnahme von Waben gibt es kein Laufen oder Abfliegen, die Bienen sitzen ganz ruhig wie bei der Carnica. Ja noch mehr - ich habe einmal beobachtet wie ein mit einem dichten Bienenpelz überzogener Kastendeckel abgelegt wurde. Während des ganzen Eingriffes in das Volk - etwa 15 Minuten - sind die Bienen auf dem Deckel vollständig ruhig sitzen geblieben. Ich würde das kaum einer anderen Rasse zutrauen.

Andererseits entwickelt aber die Biene ein ganz beachtliches Verteidigungsverhalten; schon bei kleinerer Irritation, oft bei blosser Annäherung an das Flugloch, kann es zu Massenattacken kommen. Bei vorsichtiger Arbeitsweise und geringer Rauchanwendung lassen sich aber auch diese Völker ohne Schutzkleidung stichlos behandeln.

Propolis wird von der Kretischen Biene kaum verwendet. Einmal wurde der Versuch unternommen, auf Kreta Propolis zu gewinnen. Dieser Versuch musste sehr bald ergebnislos abgebrochen werden (Chr. Zymbragoudakis). 
Der Honig wird dunkel verdeckelt. Nur ganz zu Anfang, bei guter Tracht entstehen auch weisse Zelldeckel.

Zu Räuberei neigt die Biene kaum.

An Bienenfeinden sind auf Kreta die Hornissen zu nennen. Seit die Olivenbäume aber mit Insektiziden behandelt werden, hat sich ihre Zahl stark vermindert.

Brutkrankheiten (amerikanische und europäische Faulbrut) sind vorhanden, sie bilden aber kein Problem. Hingegen tritt in den feuchten Wintern häufig Nosema in schädigender Weise auf.

Auch nördlich der Alpen lässt sich die Kretische Biene gut halten und überwintern. !. Als besonders brutfreudig und winterfest haben sich Hybriden mit Carnica-Drohnen erwiesen.

\section{DISKUSSION}

Die Festellung, dass die Kretische Biene taxonomisch keinerlei Beziehungen zu den Bienen des griechischen Festlandes oder den westgriechischen (Ionischen) Inseln aufweist, war zunächst überraschend. Von den Bienen der Balkan-Halbinsel (CarnicaGruppe) unterscheiden sich die Arbeiterinnen nach unseren Messungen in etwa 20 Merkmalen, die Drohnen in 12. Dadurch wird neuerlich bewiesen, dass eine autochthone Bienenpopulation auch durch jahrtausendelange politische und kulturelle Verbindungen nicht verändert wird.

Dagegen besteht ein sehr grosse Ähnlichkeit mit den Bienen von Anatolien, bzw. mit den Populationen von den Inseln der östlichen Ägäis.

Am deutlichsten werden die morphometrischen Beziehungen der ostmediterranen Bienenrassen auf einer Graphik, welche die Faktoren 2 und 3 gegenüberstellt, obwohl sie beide zusammen nur $40 \%$ der Gesamtvariabilität enthalten. In diesen beiden Faktoren spielen Grössenmasse kaum eine Rolle (diese sind in Faktor 1 enthalten), während Merkmale der Farbe, der Behaarung und des Flügelgeäders im Vordergrund stehen.

Dabei zeigt sich (Abb. 2), dass in dieser Darstellung eine recht gute Dreigliederung des adami-Komplexes erreicht wird (Westanatolien-ostägäische InselnKreta). Die geplante Analyse der Bienenpopulationen von ganz Anatolien wird vielleicht Aufschluss darüber geben, wie diese Situation am besten beschrieben wird, d.h. ob die westanatolischen Formen zu einer Festlandrasse mit einem grösseren Areal gehört, oder ob sie besser zu einer Inselrasse $A$. m. adami gerechnet wird. Vorderhand erscheint es trotz der angedeuteten Trennungsmöglichkeit klüger, die Bezeichnung " adami " auf die Bienen der Insel Kreta zu beschränken.

Ferner geht aus Abb. 2 hervor, dass $A$. m. adami relativ enge morphologische Beziehungen zu zwei Rassen besitzt, zu $A$. m. syriaca BuTTEL-REEPEN (Israel, Jordanien und Libanon) und zu $A$. m. intermissa Buttel-REEPEN (Tunesien und Alge- 


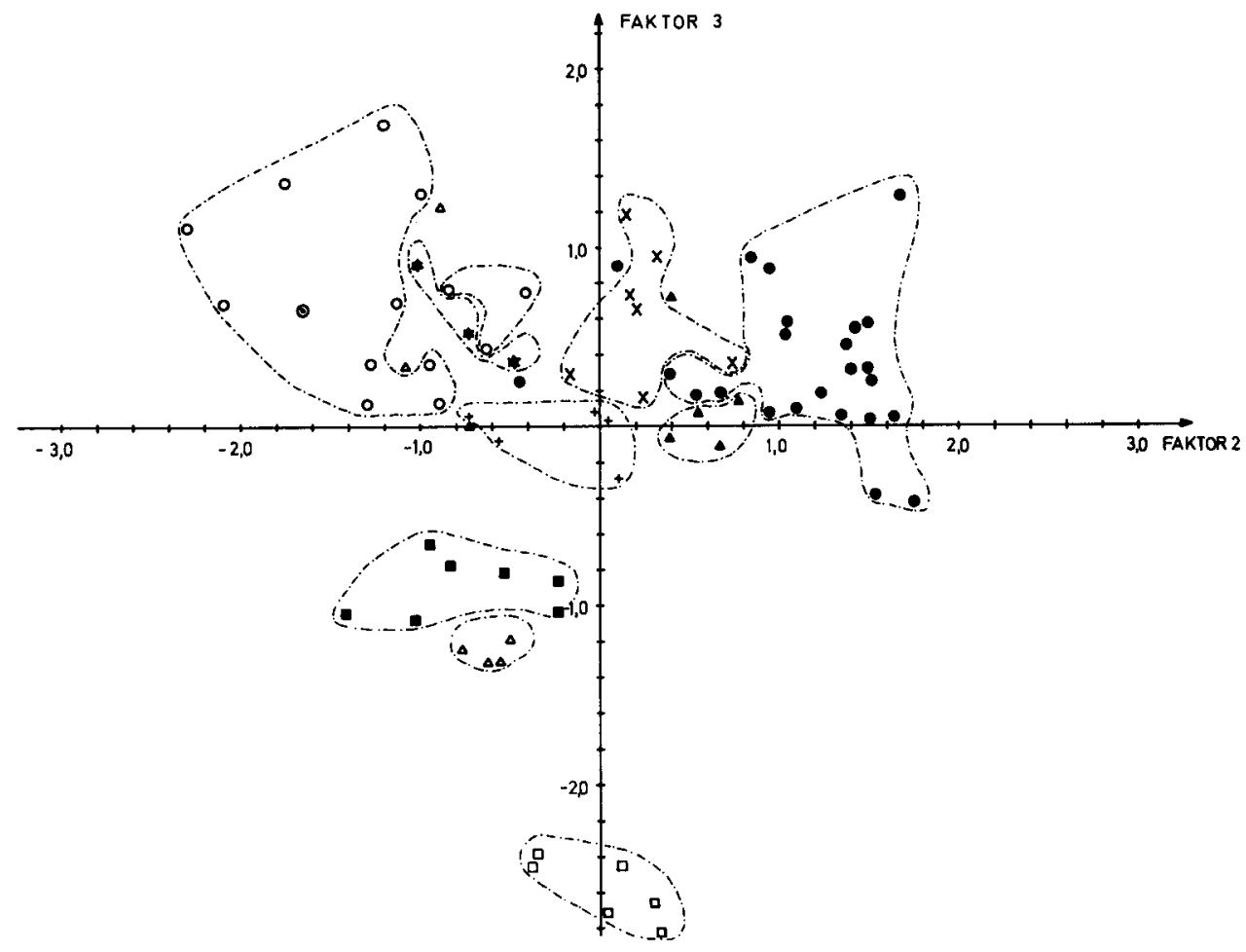

Aвв. 2. - Analyse wie in $A b b$. 1. Faktor 2 gegen Faktor 3. Relativ nahe Beziehungen von $A$. m. adami zu syriaca und intermissa. Die grösste Distanz ergibt sich zu den Proben von den Ionischen Inseln (Carnica-Gruppe). Zwischen den Bienen von Westanatolien, den ostägäischen Inseln und Kreta deutet sich morphometrisch eine Trennung an. Legende wie in Abb. 1.

FIG. 2. - Résultat de l'analyse factorielle. Facteur 2 contre facteur 3. Rapports relativement étroits d' $A . m$. adami avec syriaca et intermissa. La plus grande distance a été trouvée pour les échantillons des îles ioniennes (groupe carnica). Il existe une séparation morphométrique entre les abeilles d'Anatolie occidentale, celles des îles de la mer Égée orientale et celles de la Crète.

Légende des signes, voir Fig. 1.

rien). Damit sind auch die möglichen geographischen Beziehungen zwischen diesen Formen gegeben. Eine Klärung der morphologischen Nachbarschaft der Syrischen Biene zur Westanatolischen ist von der Untersuchung der Bienen von Südanatolien zu erwarten. Die relative morphologische Nähe der nordafrikanischen Tellbiene ist angesichts der geographischen Lage Kretas nicht überraschend.

A. m. cypria PollmanN und sicula Spinola bilden isolierte Punktschwärme. Am stärksten abgesetzt von der ostmediterranen Rassengruppe intermissa-syriacaadami sind die Proben von den Ionischen Inseln, zugehörig zur südosteuropäischen Carnica-Gruppe. 
Als eines der wesentlichen Ergebnisse dieser Untersuchung bleibt festzuhalten, dass zwischen dem griechischen Festland bzw. dem Peloponnes auf der einen und Kreta mit den ostägäischen Inseln auf der anderen Seite eine scharfe Rassengrenze besteht, in ihrer Ausgeprägtheit vergleichbar mit der Grenze zwischen den Rassen mellife ra und carnica in den Ostalpen. Solche Situationen weisen immer auf zweierlei hin :

1. Eine getrennte Entwicklung dieser Populationen seit dem frühen Pleistozän bzw. seit dem Tertiär.

2. Ein geringer genetischer Austausch zwischen den Populationen in der postglazialen Periode.

$\mathrm{Da}$ alle Rassen von $A$. mellifera $\mathrm{L}$. miteinander voll fertil hybridisieren, gibt es nur eine allopatrische Verbreitung der Rassen. Die Existenz zweier Rassen im selben Areal ist unmöglich. Berühren sich die Verbreitungsareale zweier Rassen, so wird bei eingeschränktem genetischen Austausch das eingewanderte Genom von der ansässigen Population aufgesaugt, oft ohne merkbare Spuren zu hinterlassen. Zu dieser Einschränkung des Austausches führen schon relativ niedrige Erhebungen, wie z.B. die Hügelketten der Ausläufer der Ostalpen westlich von Wien (RuTTNER, 1952). Fehlt hingegen jegliche geographische Barriere wie z.B. in Mittelrussland, so bildet sich eine breite Hybridisierungszone zwischen den Rassen (Alpatow, 1948).

Der Erstankömmling in einem bienenfreien Gebiet bleibt dann der dauernde "Platzhalter "; das erklärt die erstaunliche Stabilität einmal entstandener Verbreitungsgrenzen über lange Zeitraüme hinweg. Es bedarf massiver Eingriffe durch den Menschen im Sinne einer planmässigen Verdrängungstaktik, um eine Bienenpopulation durch eine andere $z u$ ersetzen.

Geologisch hat die Ägäis im Tertiär und im Pleistozän eine sehr bewegte Geschichte. Es gab Zeiten grosser Landmassen mit Verbindungen sowohl zum europäischen wie zum kleinasiatischen Festland und Zeiten starker Absenkung. Zumindest die grösseren Inseln, wie z.B. Kreta blieben aber immer wenigstens teilweise erhalten (Kuss, 1973, 1975).

Tiergeographisch wurden für die Inselwelt der Ägäis sowohl Beziehungen zu Peloponnes, bzw. zum griechischen Festland, wie zu Anatolien festgestellt. Dabei spielt für verschiedene rezente Reptilien und Amphibien wie für pleistozäne Säuger (Kuss, 1973) der "Karpathosgraben" zwischen Karpathos und Rhodos als Trennungslinie eine besondere Rolle. Die Inseln westlich von dieser Linie zeigen in Faunenelementen einseitige Beziehungen zum Peloponnes, östlich davon hingegen zu Anatolien.

Bei der anatolischen Form der Felsenmaus (Apodemus mystacinus) hingegen hat G. STORCH (1977) ein Verbreitungsareal gefunden, das sich von Anatolien aus über Rhodos und Karpathos bis Kreta erstreckt, also den Karpathosgraben überquert. Ein ähnliches Verbreitungsareal zeigen zwei andere auf Kreta vorkommende Säuger : Die Stachelmaus (Acomys sp.) und die Bezoarziege, Capra aegagrus (Lit. bei STORCH, 1977). 
Als weiteres Beispiel kann hier jetzt Apis mellifera adami angeführt werden, die ebenfalls auf der Linie Izmir-Karpathos-Kreta zu finden ist. Allerdings können derzeit noch keine exakten Angaben über die westliche Verbreitungsgrenze in der mittleren Ägäis gemacht werden, da bisher nur wenige Befunde vorliegen. In unserem eigenen Material befinden sich Proben von Naxos, Kithira und von den Ionischen Inseln. Während sich die Bienen von den beiden letzten Fundorten nicht von denen des südgriechischen Festlandes unterscheiden, ist die Probe von Naxos insofern bemerkenswert, als sie in einer Reihe von Merkmalen auch Beziehungen zu den Inselpopulationen der Ostägäis zeigt; auf den Graphiken der Principal-Components-Analyse liegen die Bienen von Naxos auf der Darstellung der Faktoren 1/2 mitten unter den südgriechischen Bienen, auf der Darstellung der Faktoren 2/3 hingegen deutlich abgerückt in Richtung der ostägäischen Proben.

Die auf Abb. 3 eingezeichnete Trennungslinie gibt also die nach dem gegenwärtigen Kenntnisstand vermutete Verbreitungsgrenze von $A$. m. adami an, die möglicherweise noch Korrekturen erfahren wird.

Weitgehende Unklarheit besteht über das Verbreitungsareal dieser Form in Anatolien. Nach dem oben Gesagten ist es aber wenig wahrscheinlich, dass es sich um eine Inselrasse handelt, die sekundär auf das Festland gewandert ist; viel eher dürfte die Ausbreitungsrichtung umgekehrt, vom Festland über den südägäischen Inselbogen nach Westen, verlaufen.

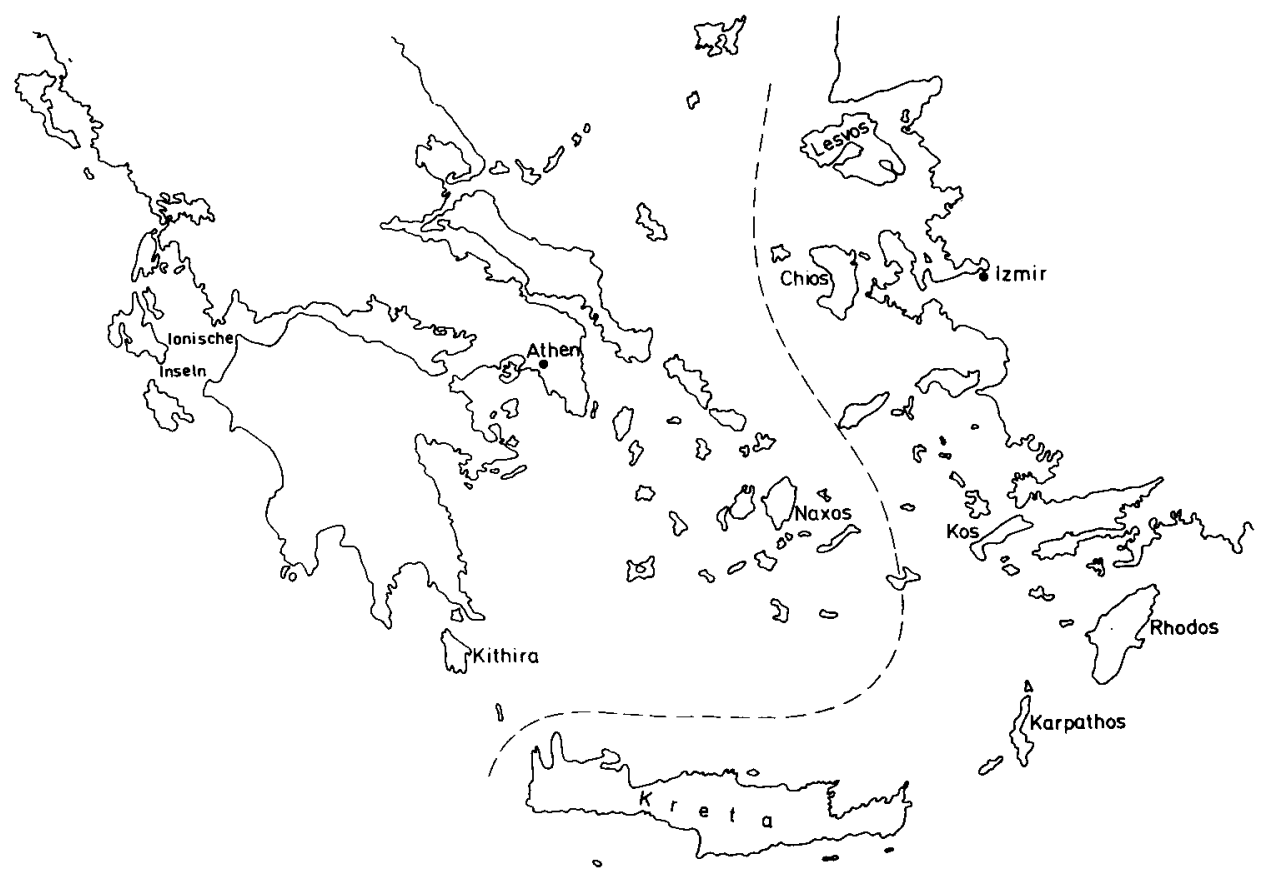

Aв8. 3. - Verbreitungsgrenze (gestrichelte Linie) von Apis mellifera adami in der Ägäis.

FIG. 3. - Limite d'extension (ligne en tirets) d'Apis mellifera adami dans la mer Égée. 
STORCH (1977) weist darauf hin, dass es sich bei den beiden von ihm untersuchten Nagern und bei der Felsenziege ökologisch um ausgesprochene Spezialisten für karge, baumlose Felslandschaften handelt, die kaum aktiv oder passiv breitere Wasserstrassen überqueren können.

Die Honigbiene fügt sich in ihren Ansprüchen exakt in diesen Biotop; in den Spalten der kahlsten Felsregionen gibt es zeitweise genügend Blütenpflanzen, um den Bienen eine ausreichende Nahrungsgrundlage zu bieten. Das zeigen die gut gedeihenden Bienen an der fast baumlosen Südseite von Kreta, sowie die autochthone Biene in dem noch viel trocknerem Bergland von Oman und Yemen (DuTton et al., 1981). Im warmen Klimabereich werden von den Bienen ohne weiteres Felsspalten anstelle von hohlen Bäumen als Nistplatz angenommen.

Über den Zeitpunkt der Besiedlung Kretas mit Bienen kann nur soviel gesagt werden, dass sie vor der postulierten Landverbindung mit dem Peloponnes (über die eine Einwanderung von Bienen aus dem Peloponnes hätte erfolgen können) stattgefunden haben muss.

\title{
DANKSAGUNG
}

Herr Christos ZymbRagoudaKis aus Chania, durch viele Jahre Bienenzuchtberater auf Kreta, war durch viele wertvolle Hinweise auf die Biologie der Biene und auf die Imkerei auf Kreta sowie durch seine Unterstützung bei der Sammlung von Proben entscheidend am Zustandekommen der Arbeit beteilligt. Bruder Adam und den Herren Tsikalas, Dr. Malitzky, Ing. SetTar und Dr. Pieper wird für die Sam-mlung von Bienenproben gedankt. A. MOHR hat in gewohnter Präzision die biometrischen Messungen durchgeführt, D. KAUHAUSEN und L. TASSENCOURT danke ich für ihre Mitarbeit bei der statistischen Analyse der Daten.

Diese Untersuchung wurde von der Deutschen Forschungsgemeinschaft durch eine Sachbeihilfe und von der Polytechnischen Gesellschaft Frankfurt am Main durch ein Reisestipendium gefördert.

Eingegangen im Juni 1980. Reçu pour publication en juin 1980.

\begin{abstract}
RESUME
Vingt-quatre échantillons d'ouvrières d'abeilles et huit échantillons de mâles provenant de diverses parties de l'île de Crète ont été analysés par une méthode standard de biométrie (RuTrNer et al., 1978). L'analyse s'applique à 34 caractères primaires concernant la taille, la pilosité, la coloration de la cuticule, les nervures alaires, ainsi que quelques indices calculés à partir de données primaires.

L'abeille crétoise est décrite comme une race géographique d'Apis mellifera propre, nettement différente des autres formes connues, ce qui confirme un travail antérieur préalable (1975). Ses caractères les plus marquants sont : une large bande tomentale, un abdomen jaune chez environ $50 \%$ des ouvrières (pigmentation homogène plus foncée chez les reines et les mâles), une taille moyenne, de larges métatarses, un abdomen large et un indice cubital faible. Par rapport aux échantillons du continent (Grèce), la plupart des caractères présentent un écart type faible.
\end{abstract}


Les abeilles d'Anatolie occidentale (Izmir) et des îles de la mer Égée orientale sont si semblables aux abeilles crétoises qu'il se peut qu'il faille les ajouter à cette race.

Les résultats de l'analyse multivariable montrent que la ressemblance est plus grande avec $A . m$. syriaca BUTTEL-REEPEN (Liban, Israël, Jordanie) et avec $A$. $m$. intermissa BUTTEL-REEPEN (Tunisie, Algérie) qu'avec les races européennes. Dans les caractères biologiques aussi (par exemple, le nombre et la situation des cellules royales d'essaimage) on retrouve des caractéristiques de l'Orient ou bien d'Afrique.

A. m. adami est une race intéressante du point de vue économique pour les conditions méditeranéennes; elle a contribué au développement remarquable de l'apiculture dans l'île de Crète. La dénomination provient du Frère ADAM, qui, le premier, a décrit l'apiculture en Crète et récolté des échantillons d'abeilles.

\section{LITERATURNACHWEIS}

Bruder ADAM, 1966. - Auf der Suche nach den besten Bienenstämmen. Ergebnisse der Rassenbewertung. Walmar Verlag, Zell Weierbach.

Alpatov V. V., 1948. - The races of honeybees and their use in agriculture (russ.). Sredi prirody, 4, Moscow Sec. Res. Nat., Moskau.

Dutton R., Ruttner F., Berkeley A., Manley M. J. D., 1981. - Observations on the Indigenous Apis mellifera of Oman. J. Apic. Res. 1981 (im Druck).

Kuss S. E., 1973. - Die pleistozänen Säugetierfaunen der ostmediterranen Inseln. Ihr Alter und ihre Herkunft. Ber. Naturf. Ges. Freiburg i. Br., 63 : $49-71$.

Kuss S. E., 1975. - Die pleistozänen Hirsche der ostmediterranen Inseln Kreta, Kasos, Karpathos und Rhodos (Griechenland). Ber. Naturf. Ges. Freiburg, 65 : 25-79.

RUTTNeR F., 1952. - Alter und Herkunft der Bienenrassen Europas. Österr. Imker, 2 : 8-12.

RUTtNer F., 1975 a. - Die Kretische Biene, Apis mellifica adami. Allg. dische Imkerztg., 9 : 271-272.

RuttNer F., 1975 b. - Die Bienenrassen Afrikas. 25. Apimondia Kongr. Grenoble, pp. 344-364.

RuttNer F., 1976. - Die Bienenzucht Kretas. Apiacta, 11 : 187-191.

Ruttner F., Tassencourt L., Louveaux J., 1977. - Biometrie und statistische Untersuchungen der geographischen Variabilität bei Apis mellifera L. 26. Apimondia Kongr. Adelaide, pp. 308-309.

RUTTNER F., TASSENCOURT L., LouveAuX J., 1978. - Biometrical-statistical analysis of the geographic variability of Apis mellifera L. Apidologie, 9 (4) : 363-381.

RUTTNER F., 1979. - Zuchttechnik und Zuchtauslese bei der Biene. Ehrenwirth-Verl., München.

STORCH G., 1977. - Die Ausbreitung der Felsenmaus (Apodemus mystacinus) : Zur Problematik der Inselbesiedlung und Tiergeographie in der Ägäis Natur und Museum, 107, 174-182. 